\title{
Construction and Characterization of EGFP Reporter Plasmid Harboring Putative Human RAX Promoter for in Vitro Monitoring of Retinal Progenitor Cells Identity
}

\section{Atefeh Atefi}

Department of Biology, Science and Research Branch, Islamic Azad University, Tehran, Iran. Department of Animal Biotechnology, Cell Science Research Center, Royan Institute for Biotechnology, ACECR, Isfahan, Iran.

\section{Pendar Shojaei kojouri}

Department of Animal Biotechnology, Cell Science Research Center, Royan Institute for Biotechnology, ACECR, Isfahan,Iran.

\section{Fereshteh Karamali}

Department of Animal Biotechnology, Cell Science Research Center, Royan Institute for Biotechnology, ACECR, Isfahan, Iran.

\section{Shiva Irani}

Department of Biology, Science and Research Branch, Islamic Azad University, Tehran, Iran.

\section{Mohammad Hossein Nasr Esfahani ( $\nabla$ nasr.royan@gmail.com )}

Royan Institute https://orcid.org/0000-0003-1983-3435

\section{Research article}

Keywords: Human retinal progenitor cells, Retinal regeneration, proliferation capacity, RAX Promoter

Posted Date: December 15th, 2020

DOl: https://doi.org/10.21203/rs.3.rs-124443/v1

License: (1) This work is licensed under a Creative Commons Attribution 4.0 International License. Read Full License

Version of Record: A version of this preprint was published at BMC Molecular and Cell Biology on August 4th, 2021. See the published version at https://doi.org/10.1186/s12860-021-00378-2. 


\section{Construction and characterization of EGFP reporter plasmid harboring putative}

2 human $R A X$ promoter for in vitro monitoring of retinal progenitor cells identity

3 Atefeh. Atefi ${ }^{1,2}$, Pendar. Shojaei kojouri ${ }^{2}$, Fereshteh. Karamali ${ }^{2 *}$, Shiva. Irani ${ }^{1}$, Mohammad

4 Hossein. Nasr-Esfahani ${ }^{2 *}$

$5 \quad$ aatefi@ royaninstitute.org

6 pendar_shojaei@yahoo.co.uk

7 *frkaramali@ royaninstitute.org

8 s.irani@ srbiau.ac.ir

$9 \quad$ * Corresponding Author:

10 "Mh.nasr-esfahani@ royaninstitute.org

$11{ }^{1}$ Department of Biology, Science and Research Branch, Islamic Azad University, Tehran, Iran

$12{ }^{2}$ Department of Animal Biotechnology, Cell Science Research Center, Royan Institute for

13 Biotechnology, ACECR, Isfahan, Iran

\section{Abstract}

15 Background: In retinal degenerative disease, progressive and debilitating conditions result in 16 deterioration of retinal cells and visual loss. In human, retina lacks the inherent capacity for 17 regeneration. Therefore, regeneration of retinal layer from human retinal progenitor cells (hRPCs) 18 is a challenging task and restricted in vitro maintenance of hRPCs remains as the main hurdle.

19 Retina and anterior neural fold homeobox gene $(R A X)$ play critical roles in developing retina and 
maintenance of hRPCs. In this study, for the first time regulatory regions of human $R A X$ gene with potential promoter activity were experimentally investigated.

22 Results: For this purpose, after in silico analysis of regulatory regions of human $R A X$ gene, the expression of EGFP reporter derived by putative promoter sequences was first evaluated in $293 \mathrm{~T}$

24 cells and then in hRPCS derived from human embryonic stem cells. The candidate region $(R A X-$ $253258 \mathrm{bp})$ showed the highest EGFP expression in hRPCs. This reporter construct can be used for 26 in vitro monitoring of hRPC identity and verification of an efficient culture medium for 27 maintenance of these cells.

28 Conclusions: Furthermore, our findings provide a platform for better insight into regulatory 29 regions of human $R A X$ gene and molecular mechanisms underlying its vital functions in retina 30 development.

31 Key words: Human retinal progenitor cells; Retinal regeneration; proliferation capacity; $R A X$

32 Promoter

\section{Background}

Retinitis pigmentosa (RP) and age-related macular degeneration (AMD) are the most common types of retinal degeneration disease (RDD) [1]. In RDD, retinal cells are damaged and thereby

36 visual ability is impaired [2]. The intrinsic regenerative capacity of the human retina is extremely

37 restricted and there is a growing focus on using human retinal progenitor cells (hRPCs) as a 38 potential therapeutic approach for restoring retinal function and visual ability [3]. In this regard, 39 RPCs can reduce disease progression rate by secretion of growth factors and upon integration and 40 differentiate into new rod and cone photoreceptors in retinal layer, can facilitate the process of 41 visual rehabilitation [4-6]. In addition, transplanted RPCs exhibit low tumorigenic potential and 
42 have tendency to differentiate into retinal cell layers [7]. However, their restricted in vitro 43 maintenance and proliferation capacity have hampered their application in the field of regenerative 44 medicine. It has been shown that they lost their proliferative ability after maximum of seven 45 passages, with reduced capacity for retinal cells formation [3]. One indirect approach to assess hRPCs preservation while being maintained in vitro, is to target their cell morphology and

47 molecular based tracking such as immunostaining. However, a more direct alternative is to use the 48 advantage of a fluorescent reporter under control of a specific retinal promoter [8]. Retina and 49 anterior neural fold homeobox gene $(R A X)$ is one of the initial genes expressed in prospective 50 retina derived from the anterior neural plate [9]. RAX transcription factor consists of two 51 conserved domains of homeodomain proteins. The first one is an octapeptide motif in the N52 terminus and the second one is a C-terminal OAR (otp, aristaless, and rax) domain, which plays 53 prominent roles in the eye development [10]. RAX is essential for the eye field specification and 54 normal development of retina. It is generally down-regulated during differentiation towards retinal 55 cells [11]. Previous researches showed positive correlation between $R A X$ expression and RPCs 56 proliferation in mouse and xenapous $[12,13]$. Human $R A X$ promoter region has not been 57 extensively examined yet [14]. In this study, after prediction of putative human $R A X$ promoter 58 regions, the expression of EGFP reporter derived by these regulatory regions was first evaluated 59 in $293 \mathrm{~T}$ cells and then in human embryonic stem cell (hESC) derived hRPCs. Distal region of 60 human $R A X$ gene containing -3097 to +161 resulted in the highest EGFP expression in hRPCs. 61 Altogether, our findings provide a better understanding of regulatory regions of human $R A X$ gene, 62 and can be extended to study the mechanism behind RAX function in multipotent retinal 63 progenitors. The identification of human $R A X$ promoter sequence might be a valuable 
64 supplementary tool for assessment of molecular pathways involved in retinal proliferation and 65 differentiation.

\section{Results}

\section{Verification of hESCs differentiation into hRPCs}

68 After differentiation of hESCs to hRPCs by RDM, the identity of hRPCs was confirmed by 69 immunocytochemical analysis of eye filed transcription factors (EFTF) panel including LHX2, 70 RAX, PAX6, SIX3 and also stemness markers like OCT4 and NANOG (Fig. 1F, G). According

71 to Figure 1, differentiation of hESCs towards retinal progenitors was verified by significant

72 reduction of OCT4 and NANOG, whereas EFTFs had successfully higher protein expression in

73 derived hRPCs. For further confirmation of proper retinal development, the relative expression of 74 EFTFs were analyzed at mRNA level by quantitative RT-PCR. Based on our results, the 75 expression of anterior neural and eye field genes including SIX3, RAX and PAX6 were increased 76 when cells cultured in RDM compared to hESCs. Moreover, the expression of stemness markers 77 including OCT4 and NANOG, were significantly decreased after differentiation of ESCs into 78 hRPCs (Fig. 1G). These results successfully confirmed the potential of selected medium for 79 differentiation of human stem cells towards retinal progenitor cells.

\section{In silico analysis of $R A X$ Promoter}

81 In this study UCSC genome browser was applied for analysis of chromatin structure (DNase 82 hypersensitivity), and chromatin state of human $R A X$ gene upstream region.

83 DNase I hypersensitivity marks different classes of cis regulatory elements within genome, such 84 as promoters and enhancers [15]. UCSC analysis showed several subset of DNase I clusters within $85 \sim 3 \mathrm{~kb}$ region upstream of human $R A X$ gene (chr18: 59273233-59276490). Also, Figure. 2A 
demonstrated the distribution of $\mathrm{H} 3 \mathrm{~K} 4 \mathrm{me} 1, \mathrm{H} 3 \mathrm{~K} 4 \mathrm{me} 3$ and $\mathrm{H} 3 \mathrm{~K} 27 \mathrm{ac}$ in 5' upstream region of $R A X$

87 gene, which are marks of active promoter and enhancer regions.

88 Based on ChIP-seq analysis in UCSC database, there are three fragments upstream of transcription

89 start site of human $R A X$ gene enriched of RNA polymerase II which can be indicator of promoter

90 activity of this region [16].

91 CpG islands are typically located near TSS and might be associated with promoter regions [17].

92 In silico analysis of upstream region of $R A X$ gene by UCSC genome browser confirmed the

93 presence of a $\mathrm{CpG}$ island of around $1 \mathrm{~kb}$ length. Moreover, these results showed high

94 evolutionarily conservation of this sequence in primates which reflects the significance of elements

95 contained in this genomic regulatory region. Collectively based on these results 3258 bp upstream

96 of human $R A X$ gene was selected as a putative region with promoter activity.

97 In order to have a better view of potential regulation of $R A X$ gene at transcriptional level, distal

98 region was also analyzed for putative binding sites of transcription factors involved in retinal

99 progenitor cell proliferation or development. For this purpose, GTRD which is a collection of

100 ChiP-seq database for identification of TFBS in human and mouse, and also JASPAR data base

101 were analyzed.

102 This sequence analysis revealed the presence of several presumptive binding sites for SOX2 and

103 OTX2 in distal region of human $R A X$ gene in accordance with previous studies in Xenopus which

104 was introduced as a conserved noncoding sequence (CNS1) by Danno et.al (Fig. 3A) [18]. UCSC

105 analysis revealed that CNS1 is highly conserved in primates and rodents (Fig. 2A). Also a number

106 of putative binding sites were predicted for SMAD2/3 within human $R A X$ gene. SMAD2/3 has

107 been introduced as a key mediator for in vitro differentiation of mouse ESCs into retinal cells by

108 direct binding to the regulatory elements of critical genes like Rax, Pax6 and Otx2 [19]. 


\section{Deletion analysis of human $R A X$ promoter}

110 To experimentally investigate the promoter activity of 5 ' flanking region of human $R A X$ gene, we 111 performed EGFP reporter assay using deletion constructs of regulatory regions. For this purpose, 112 different candidate regulatory fragments were cloned upstream of EGFP reporter (Fig .3A) and 113 transiently transfected in 293T cells (Fig. 3B, D) and hRPCs (Fig .3C, E). Based on protein atlas 114 database 293T cells exhibited a low level of $R A X$ mRNA expression. RT-PCR results also 115 confirmed the amplification of $R A X$ gene (100 bp) from CDNA synthesized from 293T total RNA 116 (data not shown). So this cell line with high transfection efficiency was selected for analysis of $117 R A X$ promoter activity. Before transfection of the putative promoter region into target cells, the 118 integrity of expression constructs was examined by restriction digestions (Fig. 2C) and sequencing 119 analysis (data not shown). Based on flow cytometry analysis $48 \mathrm{~h}$ post transfection, minimal upstream sequences of $R A X$ gene with 267 and 525 bp length resulted in low rate of EGFP expression in 293T cells. After elimination of these two minimal regions, 3007 and 2761 bp

122 fragments indicated lower EGFP expression compared to the minimal regions. On the other hand, 1231665 and 3258 bp regions could highly derive the EGFP reporter and with a similar expression 124 rate (Fig. 3B). Surprisingly, 3258 bp distal region indicated the highest $R A X$ promoter activity in 125 retinal progenitor cell and contributed to 2-fold EGFP enhancement in compared to 1665 proximal 126 promoter sequence (Fig. 3C). These results suggest that there might be enhancer elements in distal 127 region of human $R A X$ gene which might cooperate with minimal regions and thereby modulating 128 transcription of the associated $R A X$ gene.

129 Discussion

130 In retina, proliferation, differentiation and cell fate decision are among cellular events being 131 controlled by complex extrinsic and intrinsic signals [20, 21]. Transcription factors such as PAX6, 
132 SIX3, OTX2, SOX2 and RAX are intrinsic regulators of maintenance and development of RPCs $133[22,23]$.

134 In vertebrates, RAX transcription factor plays critical roles in early development of retina and has 135 been implicated in RPCs maintenance [12]. Deletion studies of Rax in mice led to loss of optic 136 vesicle development [24]. Moreover, studies have been showed that conditional knockout of $\operatorname{Rax}$ 137 resulted in failure of laminar structure formation in retina, reduction of retinal progenitor cells, and 138 retinal cell fate changes in conditional knockout mouse model [25]. However, there are still 139 ambiguous aspects of RAX transcription factor roles in mammalian eye formation.

140 Most of retinal diseases are resulted from destruction of different retinal cell types [26]. To provide 141 a sufficient pool of RPCs as a therapeutic approach for retinal degenerative disease, in vitro culture 142 of retinal progenitor cells with high capability of proliferation and multi-potency is very critical. 143 In vitro culture of ESC derived RPCs are usually led to down regulation of critical EFTFs in RPC 144 and loss of their identity over passages [27-29]. These conditions are caused to failure of providing 145 adequate pool of RPCs for downstream studies. In this study, to monitor in vitro maintenance of 146 ESCs-derived hRPCs, an expression vector driving EGFP reporter by human $R A X$ promoter was 147 designed. For this purpose, for the first time we experimentally investigated the regulatory regions 148 upstream of human $R A X$ gene with potential promoter activity.

149 First we characterized hRPCs derived from hESCs by expression analysis of eye field markers 150 [30]. In our previous studies, Noggin, IWR and IGF1 were included to the culture medium to 151 differentiation of hESCs into hRPCs [31]. In this protocol, several sequential induction steps are 152 needed to achieve retinal progenitor cells. For development of forebrain derivatives, BMP and Wnt 153 pathways should be antagonized [32, 33]. Therefore, in order to direct ESCs to the anterior neural 154 fate, EBs were treated with combination of noggin (a potent inhibitor of BMP pathway), IWR (an 

antagonist of Wnt/ $\beta$-catenin signaling pathway) and IGF-1as an inducer of retinal progenitors from ESCs under 3D culture conditions [34].

The prediction of putative promoter region was performed using different bioinformatics tools. Human $R A X$ promoter region has not been thoroughly studied so far. For this purpose, upstream flanking region of human $R A X$ gene was analyzed by UCSC in terms of chromatin state (H3K4me1, H3K4me3 and H3K27ac), DNase hypersensitive sites, CpG islands, POL II enrichment and sequence conservation [35]. DNase clusters indicated that transcriptional machinery might be enriched at these particular sequences with open chromatin structure [36]. Furthermore, our in silico analysis demonstrated the distribution of H3K4me1, H3K4me3 and H3K27ac in '5' upstream region of human $R A X$ gene. H3K4me1 and H3K4me3 epigenetic modifications are normally signature of active promoter and enhancer regions. Enrichment of $\mathrm{H} 3 \mathrm{~K} 27 \mathrm{ac}$ is also an active chromatin mark [37, 38]. Basically, RNA polymerase II binds to promoter region of genes for transcription initiation with aid of transcription factors. Most mammalian RNA polymerase II initiate transcription at $\mathrm{CpG}$ islands, which are devoid of DNA methylation [16]. UCSC analysis confirmed the presence of sites enriched by RNAP II and a $\mathrm{CpG}$ island in regulatory region of human $R A X$ gene. Collectively, $\sim 3.2 \mathrm{~kb}$ upstream of human $R A X$ gene was considered as the putative promoter region. The promoter activity of this region was experimentally investigated using deletion constructs deriving EGFP reporter. Deletion analysis of $\sim 3.2 \mathrm{~kb}$ human $R A X$ promoter regions $(-3097$ to the +167$)$ was analyzed in $293 \mathrm{~T}$ and ESCs-derived hRPCs. The results of deletion construct analysis showed that region from -3097 to $-336(R A X-2761)$ and -3097 to $-90(R A X-3007)$ of human $R A X$ gene could not independently drive downstream EGFP expression after elimination of minimal regions which basically include general regulatory binding sites required to trigger transcription. These findings indicated that the 
178 minimal $R A X$ regulatory promoter regions, from -106 to $+161(R A X-267)$ and -364 to $+161(R A X-$

179 525), consist of critical elements to derive $R A X$ gene expression. Candidate distal region $180-3097 /+161(R A X-3258)$ and proximal region $1504 /+161(R A X-1665)$ showed a significantly 181 higher EGFP expression in both 293T cells and hRPCs. Interestingly, distal region ( $R A X-3258 \mathrm{bp})$ 182 demonstrated a remarkable more EGFP expression than $R A X-1665 \mathrm{bp}$ in hRPCs. Based on our 183 results, we speculated that potential elements which are located in distal region, mediate the 184 transcriptional stimulation and contributes to the higher promoter activity of $R A X-3258$ compared 185 to $R A X-1665$.

186 Previous studies confirmed the necessity of conserved noncoding sequence 1 (CNS1) which is 187 located $\sim 2 \mathrm{~kb}$ upstream of $\operatorname{Rax}$ promoter as a regulatory region for expression of $\operatorname{Rax}$ in mice. 188 CNS1 contains a highly conserved binding sites for Sox 2 and Otx2 transcription factors across 189 vertebrates, which are required for Rax transcription in mice [18]. These studies identified that 190 Sox 2 and Otx 2 are potent modulators of Rax expression by direct binding to the promoter region 191 and synergistically activate its transcription. Interactions between Sox2 and Otx2 proteins, 192 regulate the expression of Rax during eye development [39].

193 Our bioinformatic analysis using JASPAR, GTRD and UCSC was also predicted binding sites for 194 SOX2 and OTX2 in distal regulatory region of human $R A X$ gene. This key conserved region was 195 included in RAX-3258 bp distal promoter which exhibited the most promoter activity in hRPCs 196 with high expression of SOX2 and OTX2. Interestingly, this candidate region was resulted in 197 substantial reduced expression of EGFP in 293T cells which do not express SOX2 or OTX2 198 endogenously[18]. Albeit, the roles of other transcription factors in the regulation of human $R A X$ 199 expression should not been ignored. 
200 Our in silico analysis also revealed several binding sites for SMAD2/3 in distal region of human

$201 R A X$ gene. Previous studies of in vitro differentiation of mouse ESCs into retinal cells, identified

202 SMAD2/3 as a key regulator of several retinal genes like $\operatorname{Rax}[40,41]$. Moreover, this study 203 showed that SMAD2/3 was able to directly bind to regulatory elements of retinal and 204 photoreceptor precursor genes. In fact, SMAD2/3 binds to Smad binding elements (SBEs) which 205 are located in distal promoter regions of target genes such as $R A X$ and activates their expression 206 (Fig. 4).

207 For further studies-regarding these molecular mechanisms in human, the effect of corresponding 208 transcription factor overexpression on human $R A X$ promoter activity with mutated TFBSs can be 209 investigated.

\section{Conclusion}

211 In summary, the present study introduced the regulatory region of human $R A X$ gene with high 212 promoter activity. When, this region is included in an expression vector expressing EGFP, it may 213 provide a molecular tool for monitoring retinal progenitor cell maintenance during in vitro culture.

214 Furthermore, our study can be extended towards future investigations regarding the molecular 215 mechanisms by which RAX play key roles in proliferation and development of retinal progenitor 216 cells and reveal more aspects of hRPCs regulation.

\section{Methods}

\section{Bioinformatics analysis}

219 The sequence of human $R A X$ gene was obtained from National Center for Biotechnology 220 Information (NCBI). Different bioinformatic tools were used to predict the potential promoter 221 regions of human $R A X$ gene. These software are as follows; UCSC (http://genome.ucsc.edu/), and 
222 Genomatix (https://www.genomatix.de/). Also, the putative transcription factor binding sites

223 (TFBS) within the human $R A X$ promoter region were analyzed using the gene transcription

224 regulation database GTRD (http://gtrd.biouml.org/) and JASPAR (http:// http://jaspar.genereg.net/).

225 Primers design for amplification of putative human $\boldsymbol{R} A \boldsymbol{X}$ promoter regions

226 Primers used to amplify the potential promoter regions of human $R A X$ gene were designed from

227 National Centre for Biotechnology Information (NCBI) database and confirmed by Primer-

228 BLAST and Oligo 7. The primers were reconstituted in nuclease-free water to a concentration of

$22910 \mathrm{pM} / \mu \mathrm{l}$. Then, $25 \mu \mathrm{L}$ PCR reactions containing $2 \mu \mathrm{L}$ of human genomic DNA (50 ng) as

230 template, $1 \mu 1$ of each forward and reverse primers (10 pM/ $\mu 1)$, 1X Multiplex PCR Master Mix

231 (Yekta Tajhiz Azma Co., Tehran, Iran), and $6 \mu \mathrm{L}$ of nuclease-free water were prepared,

232 Amplification was carried out in thermocycler (Thermoscientific) with amplification conditions

233 shown in Table 1.

234 Cloning of putative human $R A X$ promoter regions into pEGFP-C1 vector

235 Using the primers listed in Table 1, the candidate promoter region -3097/+161 of the human $R A X$ 236 gene, and a series of control fragments $(-106 /+161,-364 /+161,-1504 /+161,-3097 /-336,-3097 /-$

237 90) were amplified by PCR from human genomic DNA and inserted into SalI/BglII site of the 238 pEGFP-C1 vector upstream of EGFP reporter. The final expression vector was transformed into 239 E. coli DH5-alpha cells. The integrity of all target sequences was verified by sequencing before 240 evaluation of promoter activity in target cells. 
241 Table 1. List of primers for amplification and characterization.

\begin{tabular}{|c|c|c|}
\hline $\begin{array}{l}\text { Putative } \\
\text { Promoter } \\
\text { regions /Genes }\end{array}$ & Primers & Sequences $\left(5^{\prime}>3\right.$ ') \\
\hline$R A X-267$ & Sense & ATTAATAGAGAAGGGGCTGGGT \\
\hline$R A X-525$ & Sense & ATTAATTAGTCTGAAGTGAGAGG \\
\hline$R A X-1665$ & $\begin{array}{l}\text { Sense } \\
\text { Anti-sense }\end{array}$ & $\begin{array}{l}\text { GTCGACGAATTAATCAGAGGTGG } \\
\text { AGATCTCTTTGGAGACGGAGAGG }\end{array}$ \\
\hline$R A X-2761$ & Anti-sense & AGATCTCTCCCCTTGCGTTTGT \\
\hline$R A X-3007$ & Anti-sense & AGATCTACCCAGCCCCTTCTC \\
\hline$R A X-3258$ & $\begin{array}{l}\text { Sense } \\
\text { Anti-sense }\end{array}$ & $\begin{array}{l}\text { GTCGACGAATTAATCAGAGGTG } \\
\text { AGATCTCTTTGGAGACGGAGAGG }\end{array}$ \\
\hline OCT4 & $\begin{array}{l}\text { Sense } \\
\text { Anti-sense }\end{array}$ & $\begin{array}{l}\text { TCTATTTGGGAAGGTATTCAGC } \\
\text { ATTGTTGTCAGCTTCCTCCA }\end{array}$ \\
\hline NANOG & $\begin{array}{l}\text { Sense } \\
\text { Anti-sense }\end{array}$ & $\begin{array}{l}\text { CAGCTACAAACAGGTGAAGAC } \\
\text { TGGTGGTAGGAAGAGTAAAGG }\end{array}$ \\
\hline NESTIN & $\begin{array}{l}\text { Sense } \\
\text { Anti-sense }\end{array}$ & $\begin{array}{l}\text { TCCAGGAACGGAAAATCAAG } \\
\text { TTCTCTTGTCCCGCAGACTT }\end{array}$ \\
\hline SIX3 & $\begin{array}{l}\text { Sense } \\
\text { Anti-sense }\end{array}$ & $\begin{array}{l}\text { TCCTCCTCTTCCTTCTCC } \\
\text { GTTGTTGATAGTTTGCGGTT } \\
\end{array}$ \\
\hline$R A X$ & $\begin{array}{l}\text { Sense } \\
\text { Anti-sense }\end{array}$ & $\begin{array}{l}\text { CAACTGGCTACTGTCTGTC } \\
\text { CTTATTCCATCTTTCCCACCT }\end{array}$ \\
\hline PAX6 & $\begin{array}{l}\text { Sense } \\
\text { Anti-sense }\end{array}$ & $\begin{array}{l}\text { CAGCTCGGTGGTGTCTTTG } \\
\text { AGTCGCTACTCTCGGTTTA }\end{array}$ \\
\hline
\end{tabular}

243 In vitro cell culture of human embryonic stem cells

244 RH6 human embryonic stem cells (hESC, Royan institute) were seeded on $0.3 \mathrm{mg} / \mathrm{ml}$ Matrigel

245 (Sigma-Aldrich, St. Louis, MO)-coated tissue culture dishes containing DMEM/F12 medium 246 supplemented with $20 \%$ knockout serum replacement (KSR), $0.1 \mathrm{mM}$ nonessential amino acids, 2

$247 \mathrm{mM}$ L-glutamine, 1\% ITS and $100 \mathrm{ng} / \mathrm{ml}$ bFGF with daily medium exchange (Fig. 1B). 
249 The process of retinal differentiation was briefly demonstrated in Figure 1A. For neural retinal 250 differentiation, the over confluent feeder-free hESCs were dissociated and then a mechanical 251 approach was used to initiate embryoid body formation (EBs) (Fig. 1C). So hESCs were 252 transferred to low adherent dishes in order to form EBs in neural induction medium (NIM) 253 containing $1 \mathrm{ng} / \mathrm{ml}$ noggin (R\&D, 1976-NG), $3 \mu \mathrm{M}$ IWR (R\&D, 5439-DK/CF), and $5 \mathrm{ng} / \mathrm{mL}$

254 human recombinant insulin-like growth factor-1 (IGF-1) (R\&D, 291-GI) in DMEM/F12 medium 255 supplemented with 10\% KSR, $0.1 \mathrm{mM}$ nonessential amino acids, $2 \mathrm{mM}$ L-glutamine, and $1 \%$ B27 256 (Gibco, 17504- 044) for 3 days. In the next step, the EBs were dissociated by Accutase (Millipore, 257 SCR005) and were replated on $1 \mathrm{mg} / \mathrm{ml}$ laminin and $15 \mathrm{mg} / \mathrm{ml}$ Poly-L-ornithine (both from Sigma258 Aldrich) coated 6-well tissue culture plates. The culture medium was replaced with retinal 259 determination medium (RDM) containing DMEM/F12 supplemented with 1\% B27, $2 \%$ N2 260 (Gibco, 17502-048), $10 \mathrm{ng} / \mathrm{ml}$ noggin, $3 \mu \mathrm{M}$ IWR, $10 \mathrm{ng} / \mathrm{mL}$ IGF-1, and $10 \mathrm{ng} / \mathrm{ml} \mathrm{bFGF} \mathrm{as}$ 261 previously explained. This medium was exchanged every two days up to 21 days (Fig. 1D, E).

\section{Transient transfection}

263 For investigation the promoter activity of expression vectors harboring the potential promoter 264 regions of human $R A X$ gene by transfection into $293 \mathrm{~T}$ cell line, these cells were maintained in 265 DMEM supplemented with 1\% L-glutamine, $10 \%$ fetal bovine serum, $100 \mathrm{U} / \mathrm{mL}$ penicillin and $266100 \mu \mathrm{g} / \mathrm{ml}$ streptomycin and incubated at $37^{\circ} \mathrm{C}$ in $5 \% \mathrm{CO} 2$. Next equal moles of expression vectors 267 were transfected into 293T cells [42] by Lipofectamine LTX Transfection Reagent according to 268 the manufacturer's instructions (Invitrogen, Germany) in a 24 well cell culture plate. Also control 269 groups were transfected by pEGFP-C1 and promoter-less vector (pEGFP-np) for analysis the 270 efficiency of transfection and confirmation of target promoter's activity respectively. The 271 expression of EGFP reporter resulting from promoter activity of $R A X$ regulatory regions were 
272 assayed 48 hours post transfection. To explore the potential promoter activity of these regions in

273 retinal progenitors, they were also transfected into hESCs- derived hRPCs, as described above.

\section{Immunocytochemical analysis}

275 To evaluate the expression of stem cell and retinal progenitor markers, immunocytochemistry 276 staining was carried out using the primary antibodies: PAX6 (SC-11357, 1/50), RAX (LS-C53650, 277 1/200), NESTIN (ab-22035, 1/100), LHX2 (SC-81311, 1/100) and secondary antibodies Anti278 Mouse IgG-FITC (Sigma, AP124F) and Anti-Rabbit IgG-TRITC (Sigma, T6778). Briefly, 5×10 279 cell/ well coverslips coated with Matrigel were plated in 24-well plates. One day later, samples 280 were fixed with $4 \%$ paraformaldehyde for $30 \mathrm{~min}$ at room temperature. Subsequently, these cells 281 were permeabilized by $0.4 \%$ Triton $\mathrm{X}-100$ for 30 min and were stained with blocking solution282 diluted primary antibodies (BSA, $10 \mathrm{mg} / \mathrm{ml}$ ) and kept at $4^{\circ} \mathrm{C}$ overnight. Then, they were treated 283 with secondary antibodies at $37^{\circ} \mathrm{C}$ for $1 \mathrm{hr}$. Furthermore, cell nuclei were stained with DAPI (3 284 ng/ml, Invitrogen). The images were taken by fluorescent microscope (Olympus, Center Valley,

285 PA, USA) equipped with an Olympus DP70 camera.

\section{Quantitative PCR (qPCR)}

287 The total RNA of hRPCs and RH6 cells (as negative control) was extracted using RNeasy Plus 288 Mini Kit (Qiagen, Hilden, Germany). Then, cDNA was synthesized using Takara cDNA Synthesis 289 kit using random hexamer primers. All qRT-PCR reactions were performed in triplicate, and data 290 were normalized to human GAPDH mRNA. Relative fold changes in target gene expression was 291 calculated using $2^{-\Delta \Delta C t}$ method. Table 1, represented primer sequences used in quantitative PCR. 292 Moreover, to investigate the stemness state and multipotency capacity of hESCs-derived hRPCs, 293 the relative expression of several markers including OCT4, NANOG and eye field markers like 
294 NESTIN, SIX3, PAX6 and RAX were evaluated[11]. Each experiment, had a negative template 295 control (NTC) for primer specificity analysis and lack of DNA contamination. SYBR Green I 296 Master reaction mix (Thermo Fisher Scientific) was used for qPCR analysis of gene expression, 297 and amplification was detected with Light Cycler 480 ABi System.

\section{Flow cytometry analysis}

299 Flow cytometry assessment was performed to evaluate the quantification of EGFP reporter derived 300 by promoter regions of human $R A X$ gene. For this purpose, two days after transfection of target 301 cells with expression vectors, cells were detached and re-suspended in cold PBS $^{-}$and analyzed by 302 FACS Vantage flow cytometry (Becton Dickinson). Data were analyzed using BD Cell Quest Pro 303 and WinMDI 2.9 software.

\section{Statistical analysis}

305 All data were analyzed using one-way ANOVA, Tukey's post-hoc analysis and Student's t-test 306 and are shown as the mean \pm SD. In each experiment, at least three biological replicates were 307 examined. In this study, $P \leq 0.05$ was considered as statistically significant.

\section{$308 \quad$ List of abbreviations}

309 hRPCs: Human retinal progenitor cells

310 RAX: Retina and anterior neural fold homeobox

311 RP: Retinitis pigmentosa

312 AMD: Age-related macular degeneration

313 RDD: Retinal degeneration disease 
314 EFTF: Eye filed transcription factor

315 CNS: Conserved noncoding sequence

316 hESC: Human embryonic stem cell

317 TFBS: Transcription factor binding site

\section{Ethical approval and consent to participate}

319 Not applicable.

320 Consent for publication

321 Not applicable.

322 Competing interest

323 There is no competing interests to report

$324 \quad$ Funding

325 There is no funding to report.

326 Authour Contributions

327 Conceived and designed the analysis were done by MHNE \& FK, Collected the data was done

328 by AA, PSH, Performed the analysis was done by AA, PSH \& FK, Wrote the manuscript was

329 done by PSH, AA, Interpretation of the obtained information was done by MHNE \& SHI. All 330 authors read and approved the final manuscripts.

331 Acknowledgements 
We thank Dr. Kianoush Dormiani and Fatemeh Haghayegh for comments that greatly improved

the manuscript.

Author information

Department of Biology, Science and Research Branch, Islamic Azad University, Tehran, Iran

Atefeh Atefi \& Shiva Irani

Department of Animal Biotechnology, Cell Science Research Center, Royan Institute for

Biotechnology, ACECR, Isfahan, Iran

Atefeh Atefi, Pendar Shojaei Kojouri, Fereshteh Karamali \& Mohammad Hossein Nasr-Esfahani

Correspondence to Mohammad Hossein Nasr-Esfahani and Fereshteh Karamali

\section{References}

1. Gagliardi G, M'Barek KB, Goureau O: Photoreceptor cell replacement in macular degeneration and retinitis pigmentosa: A pluripotent stem cell-based approach. Progress in retinal and eye research 2019.

2. Sottile F, Pesaresi M, Simonte G, Cosma MP: Cell Therapy for Degenerative Retinal Disease: Special Focus on Cell Fusion-Mediated Regeneration. In: Cell-Based Therapy for Degenerative Retinal Disease. Springer; 2019: 217-244.

3. Young MJ, Tucker BA, Baranov PY: Low oxygen culture conditions for maintaining retinal progenitor cell multipotency. In.: Google Patents; 2017.

4. Zhou J, Benito-Martin A, Mighty J, Chang L, Ghoroghi S, Wu H, Wong M, Guariglia S, Baranov $\mathrm{P}$, Young $\mathrm{M}$ : Retinal progenitor cells release extracellular vesicles containing developmental transcription factors, microRNA and membrane proteins. Scientific reports 2018, 8(1):1-15.

5. Liu Y, Chen SJ, Li SY, Qu LH, Meng XH, Wang Y, Xu HW, Liang ZQ, Yin ZQ: Longterm safety of human retinal progenitor cell transplantation in retinitis pigmentosa patients. Stem cell research \& therapy 2017, 8(1):209.

6. Wang S-T, Chen L-1, Zhang P, Wang X-B, Sun Y, Ma L-X, Liu Q, Zhou G-M: Transplantation of retinal progenitor cells from optic cup-like structures differentiated from human embryonic stem cells in vitro and in vivo generation of retinal ganglion-like cells. Stem cells and development 2019, 28(4):258-267.

7. Singh MS, Park SS, Albini TA, Canto-Soler MV, Klassen H, MacLaren RE, Takahashi M, Nagiel A, Schwartz SD, Bharti K: Retinal stem cell transplantation: Balancing safety and potential. Progress in retinal and eye research 2020, 75:100779. 
8. Bervoets I, Charlier D: A novel and versatile dual fluorescent reporter tool for the study of gene expression and regulation in multi-and single copy number. Gene 2018, 642:474-482.

9. Kon T, Furukawa T: Origin and evolution of the Rax homeobox gene by comprehensive evolutionary analysis. FEBS Open Bio 2020.

10. Furukawa T, Kozak CA, Cepko CL: Rax, a novel paired-type homeobox gene, shows expression in the anterior neural fold and developing retina. Proceedings of the National Academy of Sciences 1997, 94(7):3088-3093.

11. Zuber ME, Gestri G, Viczian AS, Barsacchi G, Harris WA: Specification of the vertebrate eye by a network of eye field transcription factors. Development 2003 , 130(21):5155-5167.

12. Muranishi Y, Terada K, Furukawa T: An essential role for Rax in retina and neuroendocrine system development. Development, growth \& differentiation 2012, 54(3):341-348.

13. Giudetti G, Giannaccini M, Biasci D, Mariotti S, Degl'Innocenti A, Perrotta M, Barsacchi G, Andreazzoli M: Characterization of the Rx1-dependent transcriptome during early retinal development. Developmental Dynamics 2014, 243(10):1352-1361.

14. Harding P, Moosajee MJJoDB: The Molecular Basis of Human Anophthalmia and Microphthalmia. 2019, 7(3).

15. Wilken MS, Brzezinski JA, La Torre A, Siebenthall K, Thurman R, Sabo P, Sandstrom RS, Vierstra J, Canfield TK, Hansen RSJE et al: DNase I hypersensitivity analysis of the mouse brain and retina identifies region-specific regulatory elements. 2015 , 8(1):8.

16. Hampsey MJMMBR: Molecular genetics of the RNA polymerase II general transcriptional machinery. 1998, 62(2):465-503.

17. Ioshikhes IP, Zhang MQJNg: Large-scale human promoter mapping using CpG islands. 2000, 26(1):61-63.

18. Danno H, Michiue T, Hitachi K, Yukita A, Ishiura S, Asashima M: Molecular links among the causative genes for ocular malformation: Otx 2 and Sox 2 coregulate Rax expression. Proceedings of the National Academy of Sciences 2008, 105(14):5408-5413.

19. Lu AQ, Barnstable CJ: Pluripotent stem cells as models of retina development. Molecular neurobiology 2019, 56(9):6056-6070.

20. Dyer MA, Cepko CL: Regulating proliferation during retinal development. Nature Reviews Neuroscience 2001, 2(5):333-342.

21. Zhang SS-M, Fu X-Y, Barnstable CJ: Tissue culture studies of retinal development. Methods 2002, 28(4):439-447.

22. Heavner W, Pevny L: Eye development and retinogenesis. Cold Spring Harbor perspectives in biology 2012, 4(12):a008391.

23. Levine EM, Green ES: Cell-intrinsic regulators of proliferation in vertebrate retinal progenitors. In: Seminars in cell \& developmental biology: 2004. Elsevier: 63-74.

24. Medina-Martinez O, Amaya-Manzanares F, Liu C, Mendoza M, Shah R, Zhang L, Behringer RR, Mahon KA, Jamrich MJPo: Cell-autonomous requirement for rx function in the mammalian retina and posterior pituitary. 2009, 4(2).

25. Mathers $\mathrm{P}$, Grinberg A, Mahon $\mathrm{K}$, Jamrich MJN: The Rx homeobox gene is essential for vertebrate eye development. 1997, 387(6633):603-607. 
26. Ramsden CM, Powner MB, Carr A-JF, Smart MJ, da Cruz L, Coffey PJJD: Stem cells in retinal regeneration: past, present and future. 2013, 140(12):2576-2585.

27. Klassen HJ, Ng TF, Kurimoto Y, Kirov I, Shatos M, Coffey P, Young MJJIo, science v: Multipotent retinal progenitors express developmental markers, differentiate into retinal neurons, and preserve light-mediated behavior. 2004, 45(11):4167-4173.

28. Yun C, Oh J, Lee B, Lee J-M, Ariunaa T, Huh KJTe, medicine r: Generation of Retinal Progenitor Cells from Human Induced Pluripotent Stem Cell-Derived Spherical Neural Mass. 2017, 14(1):39-47.

29. Qu L, Gao L, Xu H, Duan P, Zeng Y, Liu Y, Yin ZQJSr: Combined transplantation of human mesenchymal stem cells and human retinal progenitor cells into the subretinal space of RCS rats. 2017, 7(1):1-14.

30. Amirpour N, Karamali F, Rabiee F, Rezaei L, Esfandiari E, Razavi S, Dehghani A, Razmju H, Nasr-Esfahani MH, Baharvand HJSc et al: Differentiation of human embryonic stem cell-derived retinal progenitors into retinal cells by sonic hedgehog and/or retinal pigmented epithelium and transplantation into the subretinal space of sodium iodate-injected rabbits. 2012, 21(1):42-53.

31. Cifuentes H: Induction of Human Embryonic stem cell derived retinal stem cells in vitro using transient overexpression of messenger RNA for BLIMP, ONECUT1 and OTX2. 2016.

32. Bertacchi M, Pandolfini L, D'Onofrio M, Brandi R, Cremisi FJDn: The double inhibition of endogenously produced BMP and W nt factors synergistically triggers dorsal telencephalic differentiation of mouse ES cells. 2015, 75(1):66-79.

33. Bae D, Mondragon-Teran P, Hernandez D, Ruban L, Mason C, Bhattacharya SS, Veraitch FSJSc, development: Hypoxia enhances the generation of retinal progenitor cells from human induced pluripotent and embryonic stem cells. 2012, 21(8):13441355.

34. Lamba DA, Karl MO, Ware CB, Reh TAJPotNAoS: Efficient generation of retinal progenitor cells from human embryonic stem cells. 2006, 103(34):12769-12774.

35. Laverrière J-N, L'Hôte D, Tabouy L, Schang A-L, Quérat B, Cohen-Tannoudji JJM, endocrinology c: Epigenetic regulation of alternative promoters and enhancers in progenitor, immature, and mature gonadotrope cell lines. 2016, 434:250-265.

36. Mercer TR, Edwards SL, Clark MB, Neph SJ, Wang H, Stergachis AB, John S, Sandstrom R, Li G, Sandhu KSJNg: DNase I-hypersensitive exons colocalize with promoters and distal regulatory elements. 2013, 45(8):852.

37. Pradeepa MM, Grimes GR, Kumar Y, Olley G, Taylor GC, Schneider R, Bickmore WAJNg: Histone $\mathbf{H 3}$ globular domain acetylation identifies a new class of enhancers. 2016, 48(6):681.

38. Creyghton MP, Cheng AW, Welstead GG, Kooistra T, Carey BW, Steine EJ, Hanna J, Lodato MA, Frampton GM, Sharp PAJPotNAoS: Histone H3K27ac separates active from poised enhancers and predicts developmental state. 2010, 107(50):21931-21936. 39. Danno H, Michiue T, Hitachi K, Yukita A, Ishiura S, Asashima MJPotNAoS: Molecular links among the causative genes for ocular malformation: Otx2 and Sox2 coregulate Rax expression. 2008, 105(14):5408-5413.

40. Lu AQ, Popova EY, Barnstable CJJScr: Activin signals through SMAD2/3 to increase photoreceptor precursor yield during embryonic stem cell differentiation. 2017, 9(3):838-852. 
41. Sakaki-Yumoto M, Liu J, Ramalho-Santos M, Yoshida N, Derynck RJJoBC: Smad2 is essential for maintenance of the human and mouse primed pluripotent stem cell state. 2013, 288(25):18546-18560.

42. Hornstein BD, Roman D, Arévalo-Soliz LM, Engevik MA, Zechiedrich L: Effects of circular DNA length on transfection efficiency by electroporation into HeLa cells. PloS one 2016, 11(12): 0167537.

Fig. 1: Overview of retinal differentiation and characterization of hPSC-derived RPCs. (A) Stepwise process of hPSCs differentiation into RPCs. (B) Phase contrast images of hPSCs cultured on Matrigel coated dish, (C) hPSC derived EBs cultured in low adherent dishes, (D) Expansion of RPCs from EBs on Matrigel coated dishes and (E) Expansion of hRPCs 21 days after seeding. (F) Immunocytochemistry of eye field-associated transcription factors: RAX, PAX6, LHX2 (red) and NESTIN (green). Scale bars: $100 \mu \mathrm{m}$ and $50 \mu \mathrm{m}$ (G) qPCR analysis of stemness and EFTF biomarkers in hPSCs and RPCs ( ${ }^{*} \mathrm{p}<0.05$ vs control, $\mathrm{n}=3$ ).

Fig. 2 Analysis of putative regulatory regions of human $R A X$ gene to derive EGFP reporter plasmids. (A) Screenshot from UCSC genome browser indicating human $R A X$ gene upstream region, H3K4me1, H3K4me3, H3K27ac and Pol2 patterns, CpG islands and DNase clusters. (B) Schematic representation of pEGFP-C1 plasmid in which CMV promoter was substituted with putative promoter regions of human $R A X$ gene. (C) The final expression plasmids were confirmed by restriction digestion analysis.

Fig. 3 Deletion analysis of human $R A X$ promoter regions. (A) A series of $5^{\prime}$ deletions from -3258 to +161 bp of human $R A X$ gene. $(\mathrm{B}, \mathrm{C})$ Intensity of EGFP reporter in promoter deletion constructs was determined by flow cytometry in 293T cells (B) and RPCs (C). Data are expressed as mean $\pm \mathrm{SD}(* \mathrm{p}<0.05, * * \mathrm{p}<0.005, * * * \mathrm{p}<0.0005, \mathrm{n}=3)$. (D, E) Comparison of EGFP expression derived by different $R A X$ regulatory regions in 293T cells (D) and RPCs (E) using flow cytometry and fluorescence microscopic analysis. The scale bar is $100 \mu \mathrm{m}$.

Fig. 4 TFBS analysis of human $R A X$ distal promoter region (-1665 to -3078) (A) Putative binding sites of SOX2, OTX2 and SMAD2/3 within this region were analyzed using the EPD database. (B) Profile summery of transcription factor binding sites using JASPAR data base. 


\section{Figures}

A

Day1

Day4

Day21

\begin{tabular}{l|cc|c} 
RH6 on matrigel & EBs on low adherent & Differentiation and expansion of RPCs & RPCs on matrigel \\
& Neural induction medium (NIM) & Retinal determination medium (RDM) & RDM - (Noggin//GF-I) \\
\hline
\end{tabular}
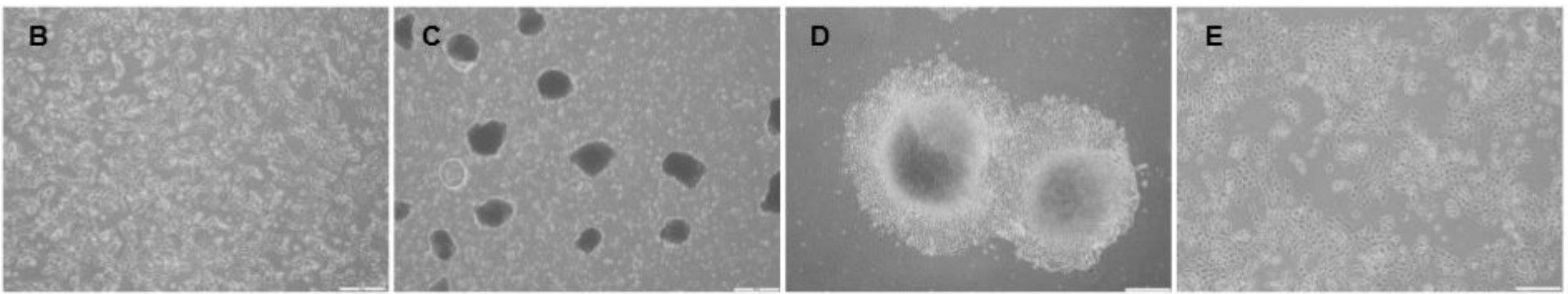

$\mathbf{F}$
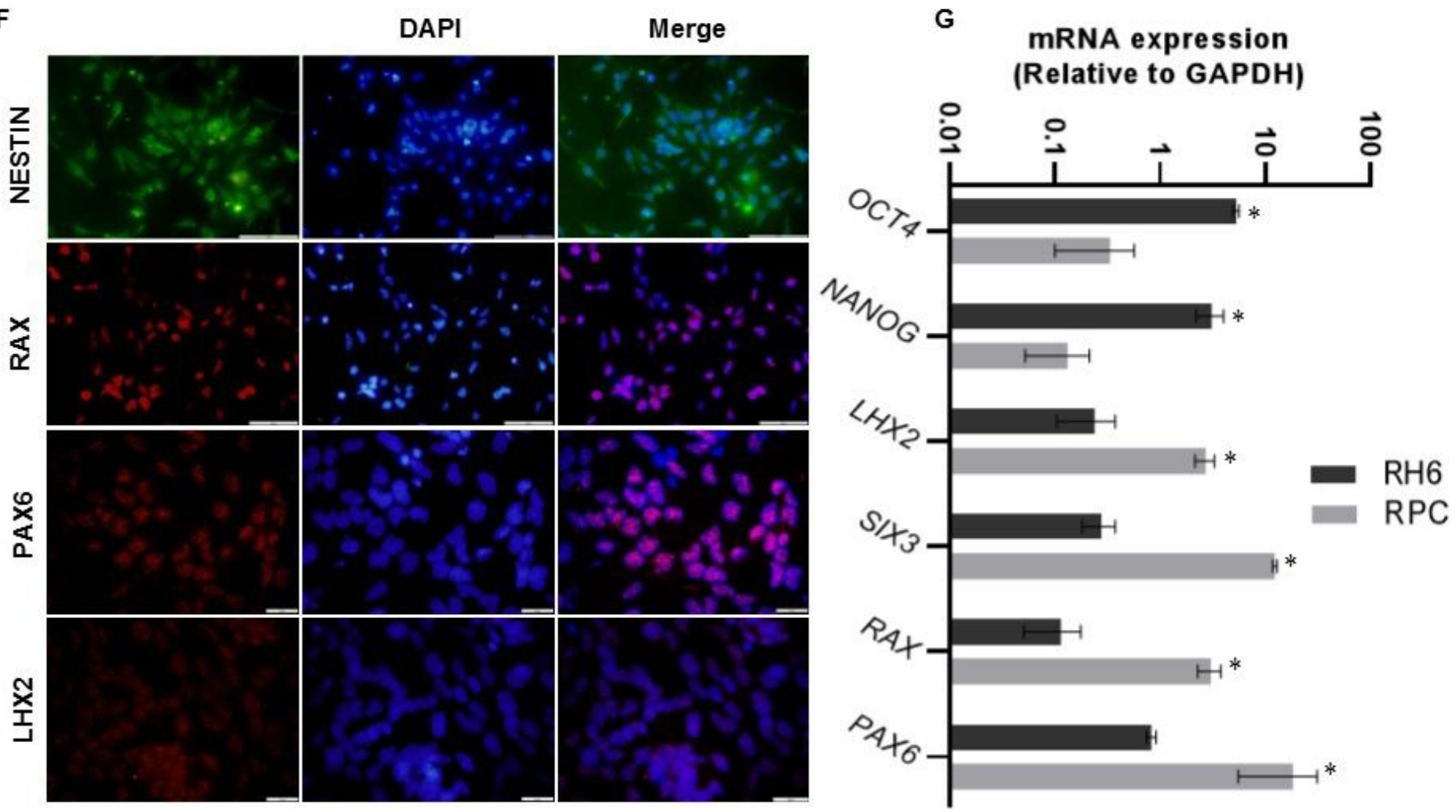

\section{Figure 1}

Overview of retinal differentiation and characterization of hPSC-derived RPCs. (A) Stepwise process of hPSCs differentiation into RPCs. (B) Phase contrast images of hPSCs cultured on Matrigel coated dish, (C) hPSC derived EBs cultured in low adherent dishes, (D) Expansion of RPCs from EBs on Matrigel coated dishes and (E) Expansion of hRPCs 21 days after seeding. $(F)$ Immunocytochemistry of eye fieldassociated transcription factors: RAX, PAX6, LHX2 (red) and NESTIN (green). Scale bars: $100 \mu \mathrm{m}$ and 50 $\mu \mathrm{m}(\mathrm{G})$ qPCR analysis of stemness and EFTF biomarkers in hPSCs and RPCs ( ${ }^{\star} \mathrm{p}<0.05$ vs control, $\mathrm{n}=3$ ). 

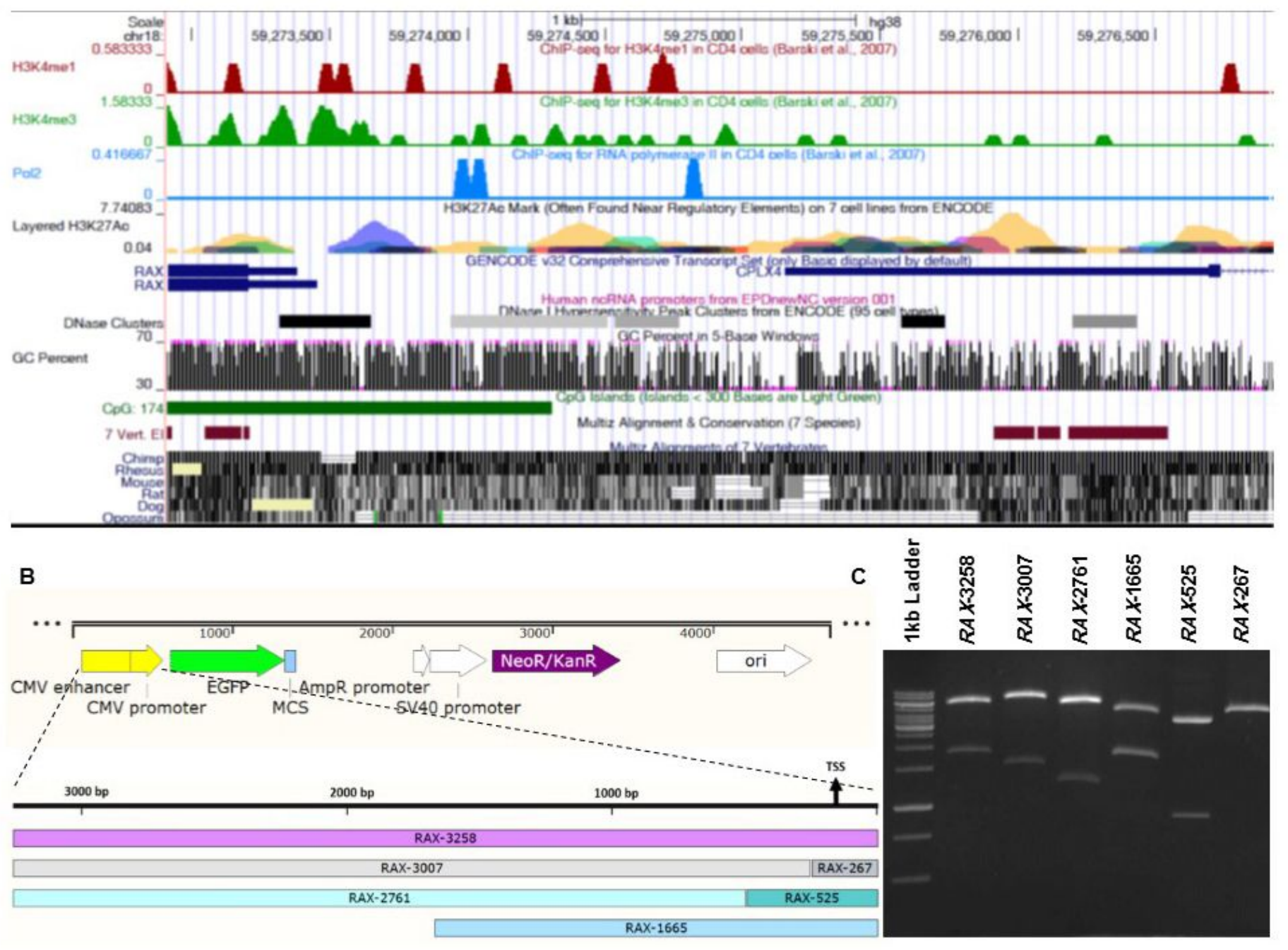

\section{Figure 2}

Analysis of putative regulatory regions of human RAX gene to derive EGFP reporter plasmids. (A) Screenshot from UCSC genome browser indicating human RAX gene upstream region, H3K4me1, H3K4me3, H3K27ac and Pol2 patterns, CpG islands and DNase clusters. (B) Schematic representation of pEGFP-C1 plasmid in which CMV promoter was substituted with putative promoter regions of human RAX gene. (C) The final expression plasmids were confirmed by restriction digestion analysis. 


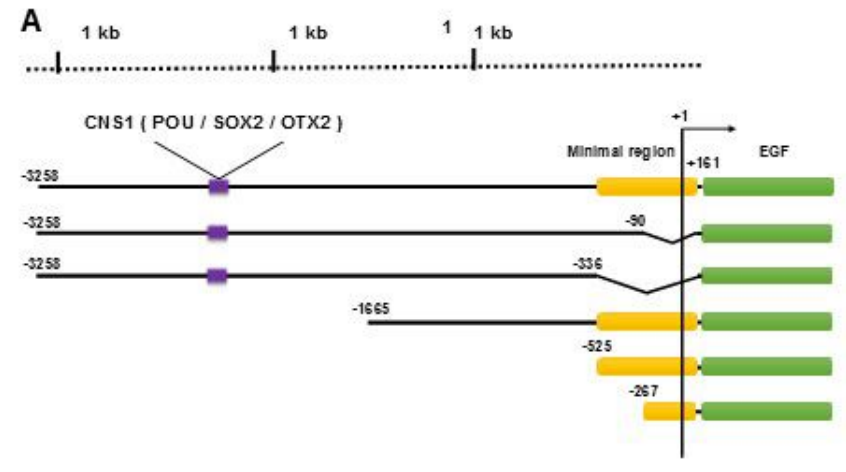

D

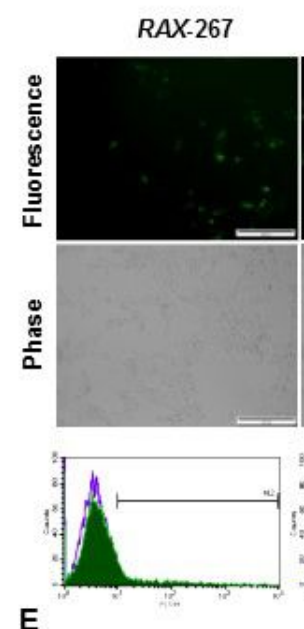

E

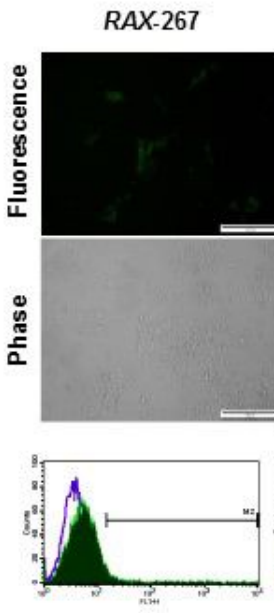

RAX-525
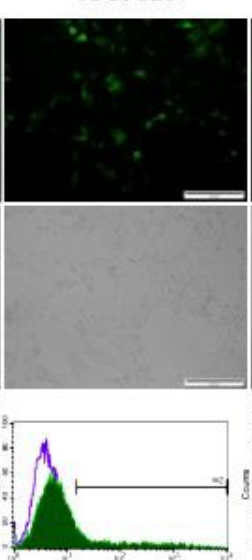

RAX-525
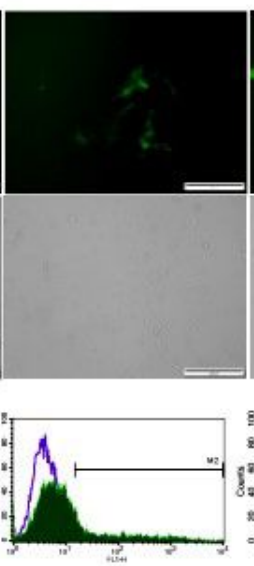

$R A X-1665$
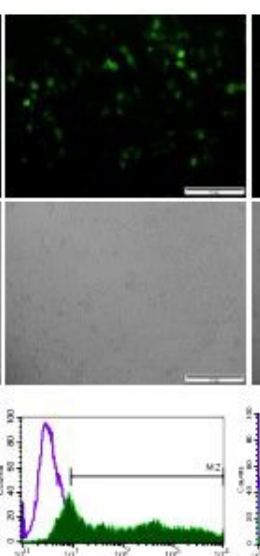

RAX-1665
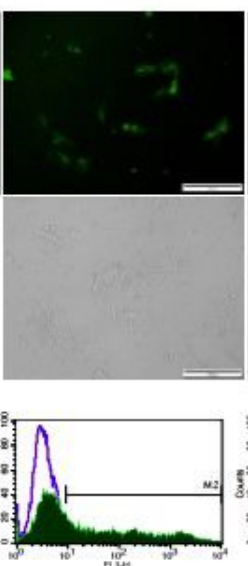

B

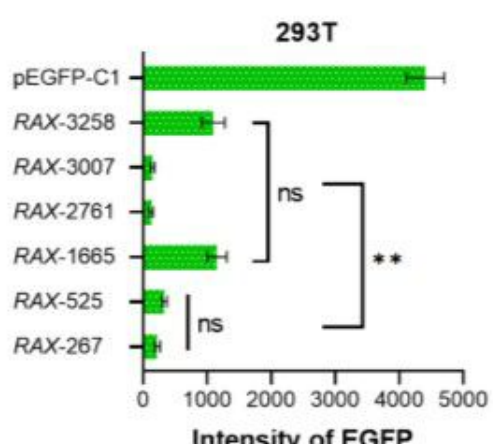

Intensity of EGFP
C

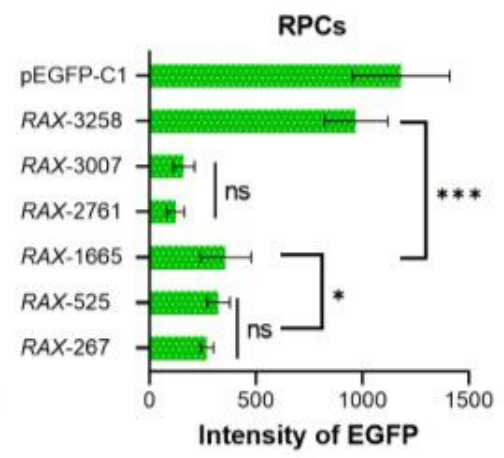

RAX-2761

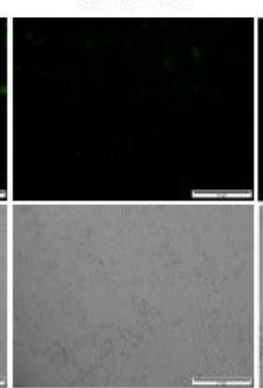

RAX-3007

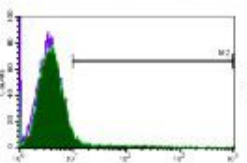

RAX-2761
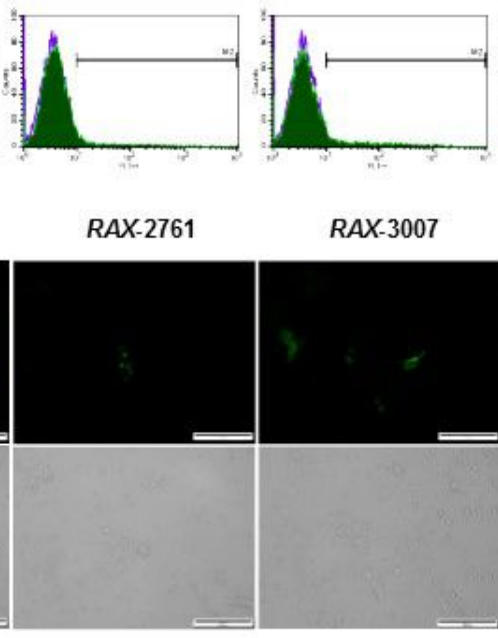

RAX-3007
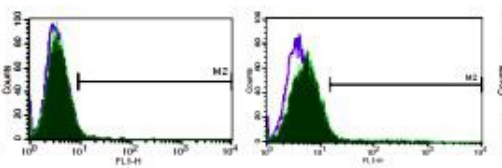

RAX-3258

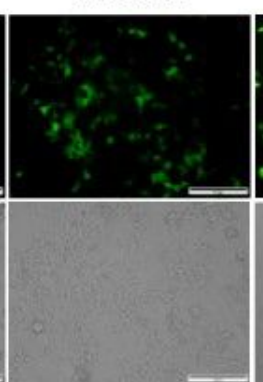

pEGFP-C1
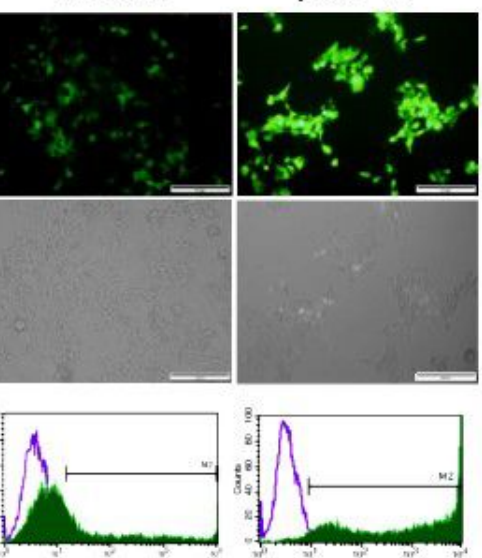

RAX-3258
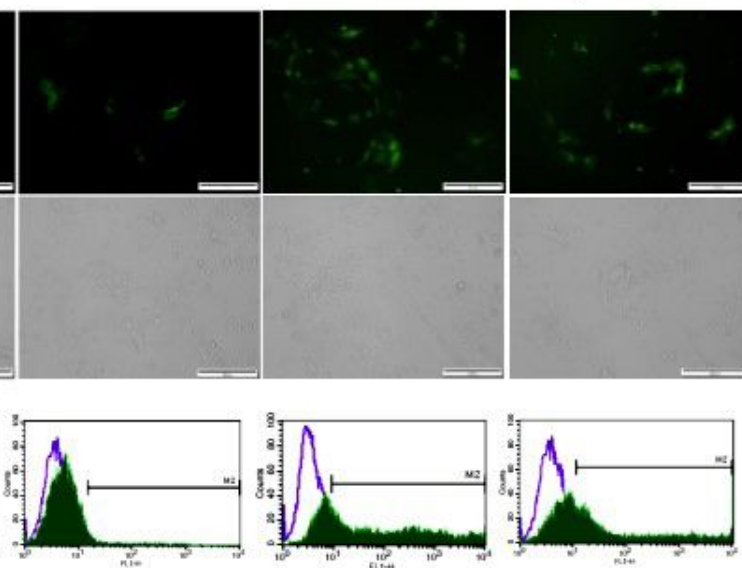

\section{Figure 3}

Deletion analysis of human RAX promoter regions. (A) A series of $5^{\prime}$ deletions from -3258 to $+161 \mathrm{bp}$ of human RAX gene. $(B, C)$ Intensity of EGFP reporter in promoter deletion constructs was determined by flow cytometry in 293T cells (B) and RPCs (C). Data are expressed as mean $\pm S D\left({ }^{\star} p<0.05,{ }^{*} p<0.005\right.$, $\star \star \star p<<0.0005, n=3)$. (D, E) Comparison of EGFP expression derived by different RAX regulatory regions in $293 T$ cells (D) and RPCs (E) using flow cytometry and fluorescence microscopic analysis. The scale bar is $100 \mu \mathrm{m}$. 
GAATtAAtCAGAgGTGGGGTTCAGAAGCCAGGCTCCTTACTGTTGCTGAAGCAGGGCTGA TTATGAGCTAGCCTAACTGCTGGAAAAATGCAGCTTCATGCTAGAAGAATTAGGGACATG GCCCAGTCCATTCATCTTCCCTAATCGAGTTCTTGGGAAATACACACGTAGATTAGCCCC TAACAATGACCCCCGGCTGATTGCTTGGAGTACCCTCCACCGTGCCCACAATGGTACTGA CTAGCTCTTTATGTCCTGCACAAAGGAAGCCCCAACTCCCCCTTGTCCTCCTCTGCCCTG TCTCCCCCACCCCCATCACCACCACCCCTGCCCTCGCCAGTGGCTGTTGAAGTCTTTTCT GCTTTTTTCCCCAGACTCTGAAACAAACAGTAAATAAAAGCTAACCTCACCTGGACTTAT TAGGAGGAGCTGGGCAGTTCCAGAGGAATTAACTTTGGAAAACAAGGCCATAAACCCCTT AGCAGCGTGTGGGCAGGAGCTTAAGGAAACGCTGCCAATCTTTGTTTAGCGGTGATTATC GAGATCAATTTAATATTGGGGAAGTTTGCATAAAAAGCATTCTCGGTCAGAGGGGGAACA ACAAATACCAATTAATTATCCCCACAGCTGCCCTCTGATCACCACCACCTCAGCACGTCA GCCACCTGGTCTATGTCACTGGCAGTCAGACCTTTTCGGGTGCCACCAGGCCATTTGTGG GCCCTGGAAAGGTGGAGGAGGGGCGGAGGAGGAGGCAAAACCTGGTCTGTCAGGGGACTT TCCAGAGGGTTTGTTAATTTGCCCTGGATGCCGGTACCCAAAGCCCACTCTCTATAGGCT CTGACTTTATTTCTTCTGGGAGCTAAACTGCTTCTACCTCCGCCTCTTAGATGCAGAATC CAGTCTCAGGGAAGGATTAAAGACAGACTGCAGGCTAAGATGGGCTTTGATTCTTTTCAA AAAGATCACACACTACAATAATAATTACAAATGAGCTGCAGGCAGAAAACACTGGACAAG GAGTCAGTGGCCTGAGTTCTAGTCCTGACCCTTCCACTTAATAGCTGTATCACTTTGAGC CTCAGTTTCCTCTTCTGTAAAATGGGGACCAATATGTCTGTCCTGCTCACCTCACAAGGT TGTGAAGATCAGATGACATGAAGAATGTGAGAAGATTCTGAAACCTACAAAGTGACATGT CGAGAGAATTGCTTGAGCCCAGGAGTTCCAGGCTGCAGTGAGCCACCATCATGCCACTGC ACTCCAGCCTGGGCAACAGAGAGGGACCCTGTCTCTAAAATAAACAGACAAATAAATAAT AAGTAAATAAAGTAATATGTGAACACTGTAAAGTGCTATTAACTTTCATTAATTTTAGCT TTTTTCTTAAAAGATAATTCTGACTTGAATTAG

\begin{tabular}{|c|c|c|}
\hline $\begin{array}{c}\text { Transcription } \\
\text { factor }\end{array}$ & Motif logo & Matrix ID \\
\hline SMAD2/3 & ACAATG CAC & MA0513.1 \\
\hline SOX2 & TAATCC & MA0143.4 \\
\hline OTX2 & MA0712.1 \\
\hline
\end{tabular}

\section{Figure 4}

TFBS analysis of human RAX distal promoter region (-1665 to -3078) (A) Putative binding sites of SOX2, OTX2 and SMAD2/3 within this region were analyzed using the EPD database. (B) Profile summery of transcription factor binding sites using JASPAR data base. 